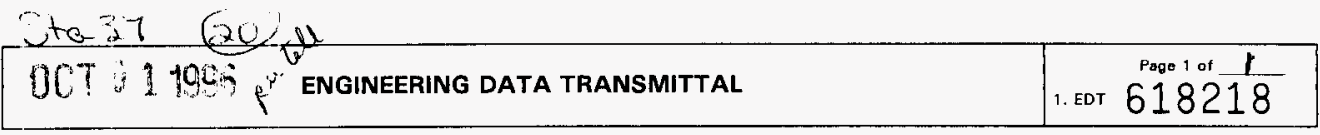

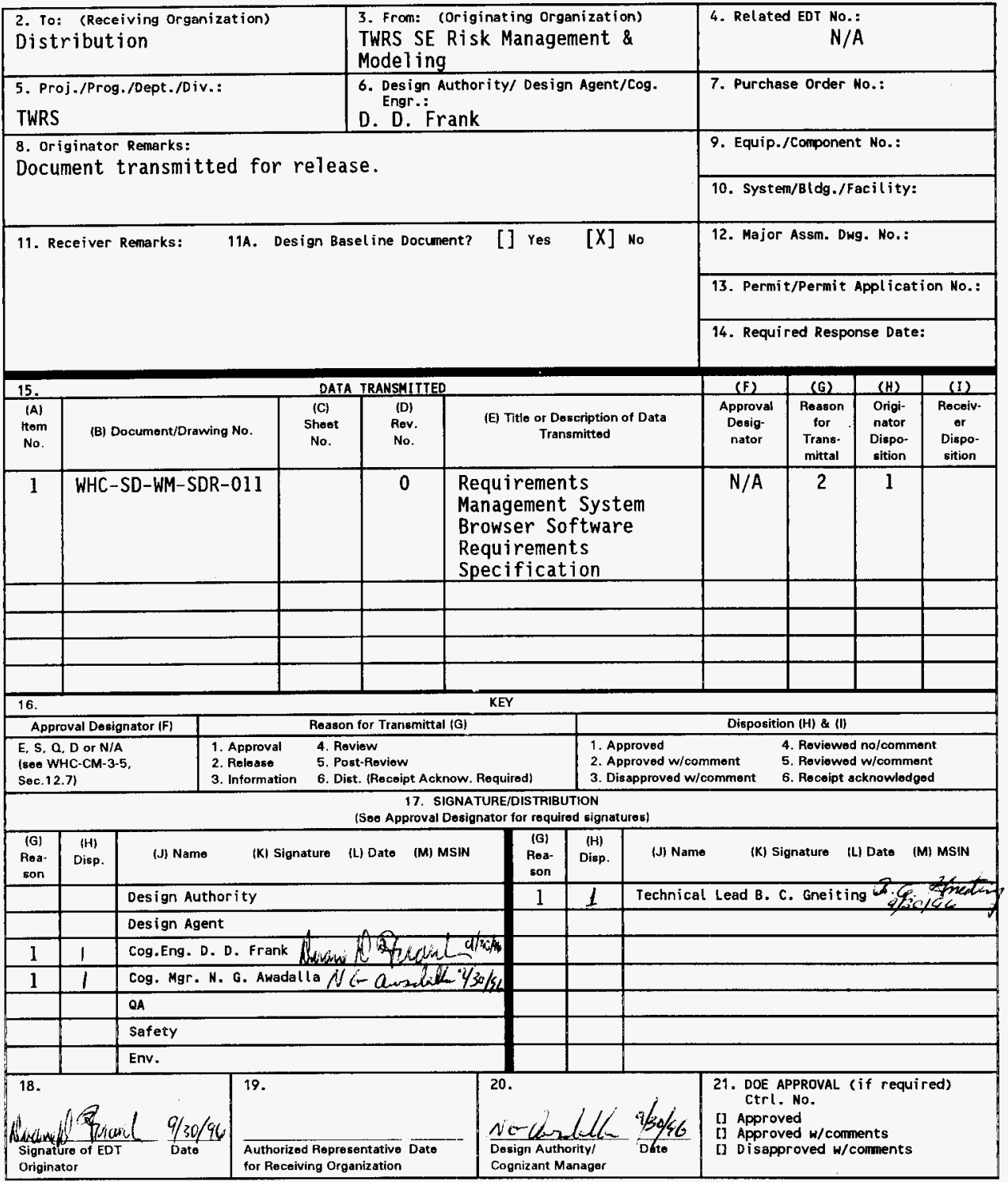

BD-7400-172-2 (05/96) GEF097 
WHC-SD-WM-SDR-011, Rev. 0

\title{
Requirements Management System Browser Software Requirements Specification
}

\author{
D. D. Frank, B. C. Gneiting
}

Westinghouse Hanford Company, Richland, WA 99352

U.S. Department of Energy Contract DE-AC06-87RL10930

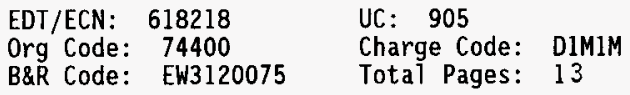

Key Words: systems engineering, requirements management, RMSB, RMACS, browser, parser, SE tools.

Abstract: The purpose of this document is to define the essential user requirements for the Requirements Management System Browser (RMSB) application. This includes specifications for the Graphical User Interface (GUI) and the supporting database structures. The RMSB application is needed to provide an easy to use PC-based interface to browse system engineering data stored and managed in a UNIX software application. The system engineering data include functions, requirements, and architectures that make up the Tank Waste Remediation System (TWRS) technical baseline. This document also covers the requirements for a software application titled "RMSB Data Loader (RMSB$D L)$ ", referred to as the "Parser." The Parser is needed to read and parse a data file and load the data structure supporting the Browser.

IRADEMARK DISCLAIMER. Reference herein to any specific commercial product, process, or service by trade name, trademark, manufacturer, or otherwise, does not necessarily constitute or imply its endorsement, recommendation, or favoring by the United States Government or any agency thereof or its contractors or subcontractors.

Printed in the United states of America. To obtain copies of this document, contact: WHC/BCS Document Control Services, P.0. 80 X 1970, Mailstop H6-08, Richland WA 99352, Phone (509) 372-2420; Fax (509) 376.4989.
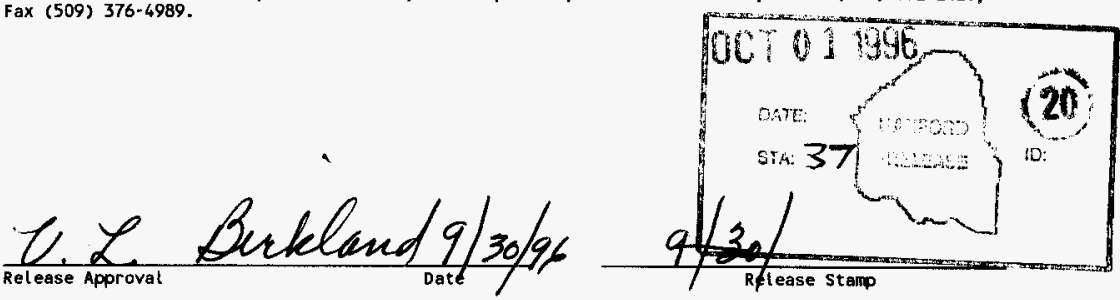
WHC-SD-WM-SDR-011 Rev. 0

\section{CONTENTS}

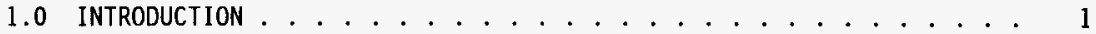

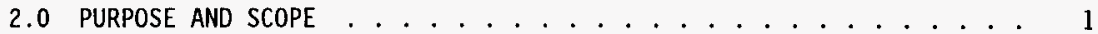

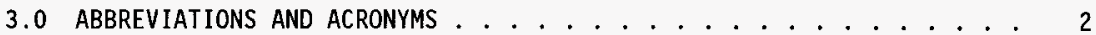

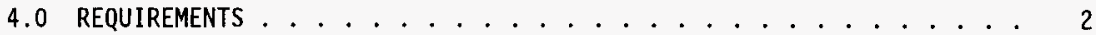

4.1 GENERAL . . . . . . . . . . . . . . . . 2

4.2 PARSER FUNCTIONS . . . . . . . . . . . . . . . . 3

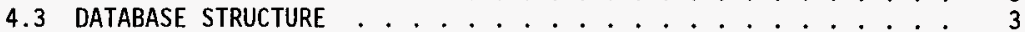

4.4 GRAPHICAL USER INTERFACE $\ldots \ldots \ldots$

4.4.1 General . . . . . . . . . . . . . 4

4.4.2 Main Window . . . . . . . . . . . . . 5

4.4.3 User Preferences Window ........... 6

4.4.4 Record Editing Window . . . . . . . . 6

4.4.5 Element Relationship Window ........... 7

4.4.6 Change Window ................ 8

4.4.7 Search Window ................ 8

4.4.8 Add Relations Window . . . . . . . . . . . . . . 9 9

4.4 .9 Change History Window .............. . 9

4.5 OTHER REQUIREMENTS . . . . . . . . . . . . . . 10

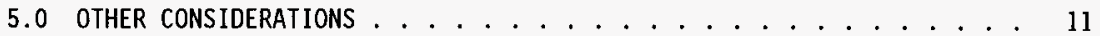

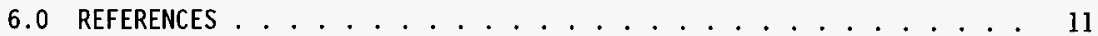




\subsection{INTRODUCTION}

Systems Engineering information for the Tank Waste Remediation System (TWRS) is currently stored and managed with the RDD- $100^{\prime}$ software application. The RDD-100 application manages data supporting the systems engineering process including functions, requirements, and architecture definitions for specifications. The RDD-100 application runs on a UNIX workstation, costs over $\$ 30 \mathrm{~K}$ per 1 icense, and is not an open system. Therefore, a personal-computer $(P C)$-based system is needed to increase the ability of engineers and managers on-site to access and modify the information stored in the RDD-100 system. To provide a more open and cost-effective access to the RDD-100 data for a wide range of users, a relational database with a graphical user interface (GUI) is required for PC users running Microsoft Windows. This software application will be titled, "Requirements Management System Browser" (RMSB) or "Browser".

The Browser data structure and interface design must closely mimic the RDD-100 data structure and terminology. The supporting database tables for the Browser shall match each element type in RDD-100 and the relationships between elements shall be recorded in a table named after the RDD-100 relationship. To transfer data from RDD-100 to the Browser, a PC-based GUI shall be developed with the title - RMSB Data Loader (RMSB-DL) or the "Parser". The Parser is required to read and parse an RDD-100 .RDT file and load the RMSB database.

\subsection{PURPOSE AND SCOPE}

The purpose of this document is to identify the software requirements for the RMSB application. This includes a specification of the database structures and the GUI. The description contains sufficient detail to describe the processes used by the software. This document does not include the RMSB installation software, or the RMSB updating software. Installation and update of the RMSB application is covered in the documentation for the TWRS Systems Engineering Tools generic installation program and generic automatic update software. Revisions of the software are covered by the draft TWRS Systems Engineering Software Configuration Management P1an

(WHC-SD-WM-CSCM-034) for the TWRS Systems Engineering Tools.

${ }^{1} \mathrm{RDD}-100$ is a registered trademark of Ascent Logic Corporation 


\subsection{ABBREVIATIONS AND ACRONYMS}

The table below identifies and defines the abbreviations and acronyms used within this document.

$\begin{array}{ll}\text { ERA } & \text { Element-Relation-Attribute, the database model used by } \\ & \text { RDD-100 } \\ \text { GUI } & \text { Graphical User Interface } \\ \text { RMACS } & \text { Requirements Management and Assured Compliance System } \\ \text { RMSB } & \text { Requirements Management System Browser, the Browser } \\ \text { RMSB-DL } & \text { RMSB Data Loader, the Parser } \\ \text { SE } & \text { Systems Engineering } \\ \text { SQL } & \text { Structured Query Language } \\ \text { TWRS } & \text { Tank Waste Remediation System }\end{array}$

\subsection{REQUIREMENTS}

The following sections identify software requirements for the development of the RMSB application. The requirements are grouped into five sections: 1) overall or general requirements for the application; 2) requirements for the utility that parses an RDD-100 data file and loads the RMSB database; 3 ) requirements for the database structure containing the data; 4) the graphical user interface requirements organized by main functionality groups (windows); and 5) other requirements that are not covered in the other four areas.

\subsection{GENERAL}

This section describes the high-level functional requirements required for the PC-based software system. The mission of this system is to provide a cost effective way for a wide spectrum of users to view the TWRS technical baseline data stored in RDD-100 and monitor changes between revisions. This bridge to RDD-100 information should also provide a platform for other applications to gain access to the system engineering data stored in RDD-100.

1. The application must run on a PC and have a Microsoft Windows type graphical user interface that operates efficientiy in the windows environment.

2. The interface must use windows, buttons, menus, scroll bars, mouseclick events, and other windows controls to manipulate the user's view of the data and to navigate through the data, i.e., a simple, easy to use, point-and-click interface.

2. The database engine storing the RDD-100 data must support the Structured Query Language (SQL) and the PC windows environment.

3. The system must be able to read an RDD-100 data file (.RDT file), parse out the required data, and download the information into a PC database structure. 
4. The database must be structured so that it mimics the RDD-100 schema and terminology to provide system commonality and ease of data transfer.

5. The graphical user interface and database structure must support the user activities of browsing, sorting, searching (data queries), monitoring changes between data sets, and allowing electronic capture and upload of set of proposed data changes made by the user.

\subsection{PARSER FUNCTIONS}

A software tool is required to initially convert the RDD-100 information, in the form of an . RDT file, into the database structure that will be used by the Browser (See Section 4.3). This tool, titled RMSB-DL or Parser is required to perform the following functions:

1. Create the database structure.

2. Parse an .RDT file generated by RDD-100, including the ability to filter out and ignore those elements, relations, and attributes not defined for the TWRS database structure.

3. Parse the change history information in the .RDT file.

4. Generate a record count report for all element and relation tables.

5. Allow the user to enter a 250 character name or description for the data set being parsed.

\subsection{DATABASE STRUCTURE}

The following requirements apply to the database structure for the Browser and the Parser applications. These requirements on the database structure conform to the schema used within RDD-100. RDD-100 uses an ElementRelation-Attribute (ERA) structure, where elements represent records of various types, the attributes are the "fields" or data values of each element record, and relations are used to identify links between elements.

1. There shall be one table for each element type used within RDD100. Elements of the same type shall be contained in a single table structure. Example of element types include Functions, Requirements, Architectures, and so on.

2. Alternate (RMACS and RDD-100) nomenclature for the elements, attributes, and relations shall be provided.

3. All of the necessary attributes associated with each element type currently used within the RDD-100 database, including the element type (String, Integer) and size (bytes) shall be retained. 
4. All of the relations currently used by RDD-100 shall be retained. Each relation shall be contained in its own table, and identify the source and target elements for the relation.

5. The data and supporting structure shall be maintained in a database engine that runs on a $P C$ in the windows environment.

6. Element records shall be indexed by the "Name" attribute, or "Number" and "Name" attributes.

7. Relation records shall be indexed by the source element type and name, and by the target element type and name.

8. Change history information for elements, relations, and attributes shall be retained in the database structure.

9. Change history information shall be indexed by the element type and element name.

\subsection{GRAPHICAL USER INTERFACE}

\subsubsection{General}

The following are requirements for the overall design and development of the GUI for the Browser software. Because the Parser will not be distributed, these requirements do not apply to the Parser. The functional requirements are generally broken down into specific groupings of functions contained in an object called a window.

1. Any window within the software that contains text input fields shall have an "Edit" menu with the standard "Cut and Paste" capabilities. These functions shall exchange data with the Windows clipboard, which can then exchange the data with other Windows applications.

2. Wherever possible, all menu items and controls (buttons, list boxes, check boxes, etc.) shall have keyboard equivalents.

3. When possible, any non-trivial windows shall have resizing capabilities, so that the controls in those windows will be sized appropriately when the window is resized by the user. This will also scale the window and controls to fit on any size screen resolution.

4. Resizable windows shall have a minimum size so that controls do not overlap and the window is still readable.

5. Resizable windows shall initially be full screen for any screen resolution greater than or equal to VGA (640 by 480 pixels).

6. All windows must be able to fit in a minimum size of a VGA monitor ( 640 by 480 pixels). 
7. For any non-trivial windows, a "Help" menu must be available with information about general topics and the current window as well.

8. Windows that contain tables of element records shall have a "double-click" event associated with them that will display the detailed information on the selected element record.

9. For any window with non-trivial information, the ability to print the information must be provided.

\subsubsection{Main Window}

The following requirements apply to the main window of the Browser. The main window shall be the initial window displayed by the application providing the starting point for the software functionality.

The Main window shall:

1. Display the "Number" and "Name" attributes for the records of a user-selected element table and provide the user withe the capability to select the element type to display from the main window.

2. Highlight element records in the main window table that have been added, modified, or deleted using various colors.

3. Provide the ability to select whether to sort the main window table by the "Name" or "Number" field.

4. Display the detailed record information for any record "doubleclicked" in the main window table. If the record is one that was deleted, a message box shall display an error message to the user. The information shall be displayed in a "Record Editing Window" described in Section 4.4.4.

5. Provide the ability to open any Browser database file.

6. Provide the ability to export the current change information into an . RDT file readable by RDD-100, or to an ASCII text file.

7. Provide access to a preferences window that allows the user to set various user preferences.

8. Provide a mechanism for exiting the program.

9. Provide a mechanism for accessing a relation tables window.

10. Provide a mechanism for accessing an old views window. 
11. Have a mechanism for adding and deleting element records in the current table.

12. Have a mechanism for accessing various query windows.

13. Have a mechanism for accessing a database changes windows.

14. Provide access to On-Line Help information.

15. Be resizable.

\subsubsection{User Preferences Window}

A preferences window shall be used to set various user defined options for the software. The window must be accessible from the main window of the software. The preferences window shall provide the user with the ability to:

1. Select and customize element views for the record editing window.

2. Select the nomenclature (labels) used by the Browser as either RDD-100 or RMACS names.

3. Select when justifications for changes to the data are made.

4. Cancel changes to the user's preferences.

5. Save the user's preferences to an initialization (.INI) file when exiting the window.

6. Provide on-line help.

\subsubsection{Record Editing Window}

A Record Editing Window shall be used to display the detailed information on an element record. This includes any and all of the attributes of the element, and any relations from the element to other elements. The user must be able to add, modify, and delete information from this window as well. Access to this window shall be by "double-clicking" on an element record reference located in other windows, including the main and search windows.

The record editing window shall:

1. Display all or a selected set of attributes for an element type as defined by the user in the Preferences window.

2. Display any or all relations to other element records in the database.

3. Allow the user to modify and save changes to the attributes. 
4. Allow the user to add a new element of the same type or delete the current element record.

5. Provide a mechanism that adds or removes relationship 1 inks to other elements.

6. Provide record navigation capabilities that navigate through records in the current element table.

7. Provide a method of canceling changes made to the attributes of the current element record.

8. Provide a mechanism for returning to the previously viewed element.

9. Provide a mechanism for accessing an old views window.

10. Provide a mechanism for viewing the change history information for the current element.

11. Provide access to on-line help for the window and current element type.

12. Be resizable.

\subsubsection{Element Relationship Window}

An element relationship or relations window shall be used to display the records in any one of the relation tables in the data structure. The relations window shall be accessible from the program's main window and have the following functionality:

1. Display the element type, name, and number for the source and target elements for a user-selected relationship type.

2. Provide a selection box for changing the relationship type being viewed.

3. Display the detailed record information for any source or target element "double-clicked" in the table.

4. Have on-line help available for the window.

5. Able to exit from the window.

6. Be resizable. 


\subsubsection{Change Window}

A change window shall be developed to display a summary of the changes for elements, relations, or attributes. Detailed information windows shall be developed to display the specifics for each type of change. Note that this is different from the change history windows, which shall show the history of changes to the master RDD-100 database. The windows that are used for displaying the change information shall be accessible from the main window.

The change window(s) sha11:

1. Allow the user to select which changes to display in the table, either elements, relations, or attributes.

2. Provide the ability to "double-click" on any change record in the main change window and display the detailed information for the selected change record. The window used to display this information will depend on the change table being viewed.

3. Allow the user to modify only the justification field in the detailed change windows; all other fields shall be automatically entered when the change is made.

4. Allow the user to exit each window.

5. Provide on-1ine help.

6. Allow the user to resize the main change window.

\subsubsection{Search Window}

The browser software shall provide one or more windows for searching through the various element tables based on the values of the attributes or relations. The user shall be able to select an instance from the search result and view the detailed information. The search windows shall be accessible from the main window of the software.

The search window(s) sha11:

1. Allow the user to query on records in a selected element table based on the attributes and/or relations associated with the elements.

2. Display a summary of all of the records found for the current search criteria.

3. Display the detailed element record information when a user "double-clicks" on that record of the results.

4. Provide a method of clearing the search results and starting over. 
5. Provide a mechanism for exiting the windows.

6. Provide $0 n-1$ ine help.

\subsubsection{Add Relations Window}

An add relations window shall be developed that provides the user the ability to select and add relations from a source element to one or more target elements. The add relations window shall be accessible from the record editing window.

The add relations window shall:

1. Allow the user to select a target element type for adding relations.

2. Allow the user to select a specific relation when more than one relation is used between the current element type and the selected target element type.

3. Allow the user to select one or more elements to add as the targets of the selected element relationship.

4. Provide a means of canceling a relationship addition.

5. Provide a means of exiting the window and adding a relation.

6. Have on-line Help.

7. Be resizable.

\subsubsection{Change History Window}

Change history windows shall be developed to display any of the changes associated with an element record. The change history will be an archive of all of the changes made to the master RDD- 100 baseline since the first revision. The windows shall allow the user to compare various versions of text attributes and identify the differences. Note that this is different from the change windows, which display only the changes to the current data set. The change history windows shall be accessible from the record editing window.

The change history window(s) sha11:

1. Display all of the element, attribute, and relation changes to a current element record.

2. Allow the user to select any versions of an attribute value from the history for comparison. 
3. Display "redline" formatted text to identify the differences between previous versions of the data set for text values. The user must be able to print this "redline" text information.

4. Have a mechanism for exiting the windows.

5. Have on-line Help information available.

6. Be resizable.

\subsection{OTHER REQUIREMENTS}

This section contains miscellaneous requirements that do not fall into one of the four categories above.

1. Changes to the database information shall be verified for correctness before they are allowed. Example: A "Name" attribute may not be blank, a "Number" attribute must be in the correct form for a hierarchical number.

2. When exporting or saving to a file that already exists, the user must give permission before the file is overwritten.

3. Database errors must be trapped and error messages displayed to the user. Example: Field too long or a string entered when a numeric value is expected.

4. The minimum platform for the application will be a $386 \mathrm{PC}$ running Windows with at least $8 \mathrm{MB}$ of memory.

5. The on-line help must provide a point-of-contact for problems that are not solved by the on-line help.

6. The software shall provide a mechanism for identifying the current program and data versions.

7. The on-line help shall provide information on each non-trivial windows in the application.

8. The on-line help shall provide information about the underlying database structure.

9. The software shall provide a way for identifying the user making proposed changes to the baseline data by querying for and storing a user name during first time use. 


\subsection{OTHER CONSIDERATIONS}

This section presents other considerations and requirements that are typically addressed separately in WHC-CM-3-10, Software Practices, Appendix F.

Functional Requirements: The functional requirements are covered in Section 4 above.

External Interface Requirements: There are no additional hardware, software, or communication interfaces for the Browser or Parser software.

Performance Requirements: As mentioned in Section 4.5, Requirement 4 , the software must run on a $386 \mathrm{PC}$ running Windows with $8 \mathrm{MB}$ of memory. This was chosen as the "Towest common denominator" for PCs onsite.

Design Constraints: None.

Attributes: None.

0ther Requirements: Testing and Acceptance criteria and documentation requirements are 1 isted in the Configuration Management Plan. Training will be addressed as needed. 0ther sections in Software Practices manual for defining potential requirements are not considered applicable.

\subsection{REFERENCES}

WHC-CM-3-10, Software Practices, Revision 0, Appendix F, "Software Requirements Specifications," Westinghouse Hanford Company.

WHC-SD-WM-CSCM-034, TWRS Systems Engineering Software Configuration Management Plan, Draft Revision, dated September 1996. 


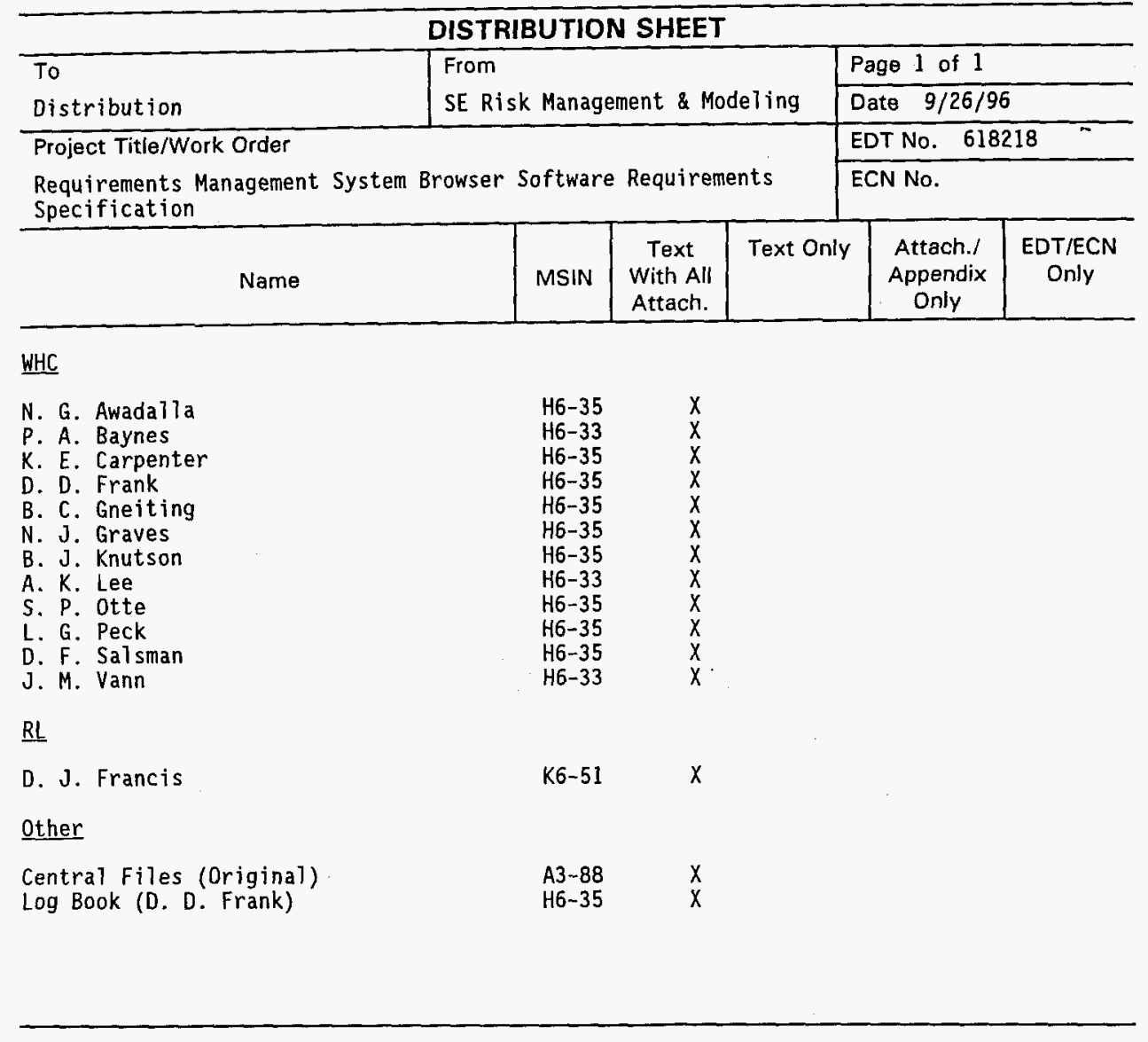

